\title{
Molecular Profiling of Human Primary Chondrosarcoma-Derived Spheres Reveals Specific and Target Genes Involved in Multidrug Resistance and Metastasis
}

Vincenzo Desiderio ${ }^{1,2 \#}$, Francesca Paino ${ }^{1 \#}$, Angela Nebbioso ${ }^{3}$, Lucia Altucci ${ }^{3,4}$, Giuseppe Pirozzi ${ }^{5}$, Federica Papaccio ${ }^{1}$, Marcella La Noce ${ }^{1}$ Alfredo De Rosa ${ }^{6}$, Gianpaolo Papaccio ${ }^{1 \star \S}$ and Virginia Tirino ${ }^{1 \S}$

${ }^{1}$ Department of Experimental Medicine Section of Histology, TERM Laboratory 2nd University of Naples, Naples, Italy

${ }^{2}$ Department of Otolaryngology: Head and Neck Surgery, University of Michigan Ann Arbor, Michigan, USA

${ }^{3}$ Department of Biophysics, Biochemistry and General Pathology, 2nd University of Naples, Naples, Italy

${ }^{4}$ Institute of Genetics and Biophysics (IGB) "Adriano Buzzati Traverso", Naples, Italy

${ }^{5}$ Department of Experimental Oncology, National Cancer Institute, Naples, Italy

${ }^{6}$ Department of Medicine, Surgery and Dentistry, 2nd University of Naples, Naples, Italy

"These authors contributed equally to this work

$\S$ These authors are equal senior authors

\begin{abstract}
Chondrosarcoma is a malignant bone tumor that accounts for approximately $25 \%$ of all bone neoplasms. The "Cancer Stem Cell" (CSC) hypothesis states that tumor contains a cell subpopulation with stemness features. Sphere cultures are routinely used for self-renewal assays and to select CSCs. Our purpose was to investigate the gene profile of chondrospheres and identify target genes for chondrosarcoma treatment. Whole-genome microarray was used to compare the gene expression of floating spheres with that of adherent counterparts derived from a human primary chondrosarcoma. In addition, CD133, OCT4, SOX2, and collagen type II markers were tested both with Real-Time PCR and flow cytometry, and cell cycle analysis and resistance to cisplatin treatment were performed. Microarray analyses revealed that 1405 genes were found differentially expressed, of which 629 genes were upregulated and 776 down-regulated in chondrospheres, with a 2 -fold cut-off threshold. Restricting analyses with a 3 -fold cut-off threshold, the number of up-regulated and down-regulated probes was 251 and 302, respectively. The most highly up-regulated genes were involved in stemness, multidrug resistance, cell cycle, apoptosis regulation, migration, motility, and invasion. Furthermore, chondrospheres expressed CD133, OCT3/4, and SOX2, and showed a remarkable resistance to cisplatin-induced apoptosis compared with their adherent counterpart. In conclusion, this study highlights that: (i) the molecular profile of chondrospheres identifies genes that are potential targets for chondrosarcoma treatment and (ii) chondrospheres are strongly resistant to cisplatin treatment.
\end{abstract}

Keywords: Chondrospheres; Cancer stem cells; Gene expression; Multi-drug resistance; Metastasis

\section{Introduction}

Chondrosarcoma is a malignant, cartilage-forming bone neoplasm that accounts for approximately $25 \%$ of all primary bone tumors. It is the third most common primary malignant bone tumor after myeloma and osteosarcoma. Typically low grade, these neoplasms can arise either de novo or from pre-existing cartilage lesions, such as an osteochondroma or an enchondroma.

It is generally believed that chondrosarcomas are relatively resistant to conventional therapies because of their extracellular matrix composition, low percentage of dividing cells, and poor vascularity [1]. Thus, surgical resection has been the main treatment for over 50 years [2]. However, several novel therapeutic approaches have been evaluated in recent years [3-5].

Unfortunately, a minority of patients presents with recurring metastases, and up to $13 \%$ of recurrent chondrosarcomas are of a higher grade than the original neoplasm [6]. There is no consensus on prognostic factors that determine which patients have a higher risk of treatment failure and dis $\neg$ ease-related death, although several papers have addressed this issue $[7,8]$. One reason for this may be that most studies were conducted on patients treated over several decades and did not account for the different surgical criteria, indications, and methods applied over the years. Furthermore, most stud-ies were done with only short follow-up - despite the fact that a high rate of late recurrence and metastases has been reported for chondrosarcoma patients compared with those with other primary bone sarcomas [9] and on patients presenting with rare histopathological sub $\neg$ types that have a distinct biologic behavior $[1,10]$ such as dedif $\neg$ ferentiated chondrosarcoma, mesenchymal chondrosarcoma, and clear cell chondrosarcoma thus reducing the validity of the results.

There is increasing evidence that many cancers are constituted by a hierarchy of cells, including so-called Cancer Stem Cells (CSCs). CSCs are believed to be progenitor cells from which the tumor is spawned and that may be responsible for relapses and metastases [11]. These

*Corresponding authors: Prof. Gianpaolo Papaccio, MD, PhD, Department of Experimental Medicine, Section of Histology, TERM Laboratory, 2nd University of Naples, Head of Cancer Stem Cells Eradication Program, via L. Armanni 5, 80138 Naples, Italy, Tel: +39 081-5666014; Fax: +39 081-5666015; E-mail: gianpaolo.papaccio@unina2.it

Received August 05, 2013; Accepted October 16, 2013; Published October 23, 2013

Citation: Desiderio V, Paino F, Nebbioso A, Altucci L, Pirozzi G, et al. (2013) Molecular Profiling of Human Primary Chondrosarcoma-Derived Spheres Reveals Specific and Target Genes Involved in Multidrug Resistance and Metastasis. J Carcinogene Mutagene $\mathrm{:} \mathrm{:} \mathrm{152.} \mathrm{doi:10.4172/2157-2518.1000152}$

Copyright: (c) 2013 Desiderio V, et al. This is an open-access article distributed under the terms of the Creative Commons Attribution License, which permits unrestricted use, distribution, and reproduction in any medium, provided the original author and source are credited. 
Citation: Desiderio V, Paino F, Nebbioso A, Altucci L, Pirozzi G, et al. (2013) Molecular Profiling of Human Primary Chondrosarcoma-Derived Spheres Reveals Specific and Target Genes Involved in Multidrug Resistance and Metastasis. J Carcinogene Mutagene 5: 152. doi:10.4172/21572518.1000152

cells have a particular ability to grow as spheres when cultured in suspension in serum-free medium supplemented with growth factors and other compounds. This ability was first described for the expansion of neuronal stem cells by Reynolds in 1992 [12].

Sphere culture has been increasingly used as method for enriching stem cells and relies on their property of anchorage-independent growth. Studies have reported the application of sphere culture to isolate, enrich, maintain, or expand potential CSC subpopulations from various types of cancers [13-16]. Like stem cells, tumor sphereforming cells are capable of proliferation and self-renewal, and possess higher tumorigenicity. Using a sphere formation system, Hansford et al. [17] for the first time successfully expanded tumor cells both from neuroblastomas and metastases of high-risk tumors. The spherederived cells formed metastatic tumors in a murine xenograft model with as few as 10 cells and could also be serially passaged [17].

We have recently reported that $\mathrm{CD}_{133^{+}}$cells from stabilized osteosarcoma cell lines possess stemness features, such as a potential for differentiation, a high proliferation rate, an ability to form osteospheres and colonies on soft agar, a capacity to exclude the fluorescent DNAbinding dye Hoechst 33342, and the expression of high levels of ABCG2, a multi-drug resistance protein of the $\mathrm{ABC}$ transporter family [18]. Moreover, in primary chondrosarcoma and osteosarcoma cell lines from human biopsies, we found $\mathrm{CD}_{133^{+}}$subpopulations displaying a capacity to grow as spheres: these spheres initiated and sustained tumor growth in immunocompromised mice, expressed stemness genes, such as OCT3/4, NANOG, SOX2, and nestin, and differentiated into mesenchymal lineages, such as osteoblasts and adipocytes [19].

Therefore, because chondrospheres are enriched in cancer stem cells, we decided to compared the molecular expression profile of chondrospheres obtained from a primary chondrosarcoma cell line with the corresponding adherent counterpart. We found that chondrospheres over-express genes involved in stemness, drug resistance, tumorigenesis, cell death, and metastasis. Specifically, $C R O P 1$, as well as TAP1 genes, involved in multi-drug resistance were up-regulated on chondrospheres. Again, VEGFA and MALAT1 genes, involved in tumor angiogenesis and metastasis formation, resulted to be over-expressed. Taken together, all these observations suggest that these genes could be potential target genes for the development of specific strategies for chondrosarcoma treatment.

\section{Materials and Methods}

\section{Patient and generation of a primary cell line}

The chondrosarcoma cell line used was obtained in our laboratory from a consenting, 59-year-old male chondrosarcoma patient attending the National Cancer Institute of Naples, Italy. Diagnosis was based on clinical and histological parameters according to WHO classification. The site of tumor involvement was the sternum. The resected tumor measured $18 \times 15 \times 8 \mathrm{~cm}$, was graded G2, focally G3, and was characterized by hypercellularity, the presence of binucleated cells, and elongated, hyperchromatic nuclei. Survival from diagnosis was 8 months. The patient eventually presented extraskeletal metastases and died of chemotherapy-related complications.

The tumor biopsy was dissected, minced, and digested with $1 \mathrm{mg} /$ $\mathrm{ml}$ collagenase I (Invitrogen Life Technologies, San Giuliano Milanese, Milan, Italy) and $1 \mathrm{mg} / \mathrm{ml}$ dispase at $37^{\circ} \mathrm{C}$ overnight with intermittent shaking. Any isolated cells were washed with PBS twice and filtered through a $70 \mu \mathrm{m}$ filter to generate a stabilized cell line. Cells were cultured in DMEM supplemented with $2 \mathrm{mM}$ glutamine, $100 \mathrm{IU} / \mathrm{ml}$ penicillin, $100 \mu \mathrm{g} / \mathrm{ml}$ streptomycin, and $10-$ or $20-\%$ heat-inactivated FBS (Invitrogen Life Technologies) at $37^{\circ} \mathrm{C}$ in a humidified atmosphere under $5 \% \mathrm{CO}_{2}$ until an adherent, homogeneous cell population was obtained. The medium was changed twice a week.

\section{Chondrosphere culture}

Primary chondrosarcoma cells were plated at a density of $1.0 \times 10^{6}$ cells $/ 25 \mathrm{~cm}^{2}$ in ultra-low-attachment flasks (Corning, Corning, NY, USA) and cultured in condrosphere medium consisting of DMEM/F12 supplemented with progesterone $(10 \mathrm{nM})$, putrescine $(50 \mu \mathrm{M})$, sodium selenite $(15 \mathrm{nM})$, transferrin $(13 \mu \mathrm{g} / \mathrm{ml})$, insulin $(10 \mu \mathrm{g} / \mathrm{ml})$, human epidermal growth factor (EGF; $10 \mathrm{ng} / \mathrm{ml}$ ), and human basic fibroblast growth factor (bFGF; $10 \mathrm{ng} / \mathrm{ml}$ ) (all from Sigma, St. Louis, MO, USA). Fresh aliquots of EGF and bFGF were added every day. After culture for 48-72 hours, chondrospheres were visible under an inverted phasecontrast microscope (TS 100; Nikon, Milan, Italy). The medium was changed twice weekly to renew the growth factors.

\section{Flow cytometry}

Chondrospheres and adherent cells (at a density of $3 \times 10^{5}$ cells/ sample) were incubated with fluorescent-labeled monoclonal antibodies or respective isotype controls. The antibodies used were anti-CD133/2PE (Miltenyi Biotec, Calderara di Reno, Bologna, Italy), anti-OCT4-PE, anti-SOX2-FITC (all purchased from BD Pharmingen, Milan, Italy), and anti-collagen type II (COL2; AbCam, Cambridge, UK). Secondary antibody was anti-rabbit FITC (AbCam). For CD133, the antibody was incubated for $30 \mathrm{~min}$ at $4^{\circ} \mathrm{C}$ in the dark. After washing steps, the cells were analyzed. For intracellular staining of OCT4, SOX2, and COL2, cells were processing using the Fix \& Perm Kit (Invitrogen, Milan, Italy) following the manufacturer's guidelines. All data were acquired using FACS Aria II (BD) and analyzed using FCS version 3 software.

\section{Cisplatin treatment}

Chondropheres and adherent cells were exposed to different concentrations of cisplatin for $48 \mathrm{~h}$. The concentrations tested were 30-, 50-, 70-, 100-, and 150- $\mu \mathrm{M}$. After treatment, chondrospheres and adherent cells were analyzed for apoptosis and cell cycle. Cell death was evaluated with flow cytometry using the Annexin/PI kit (Invitrogen) according to the manufacturer's guidelines and the data analyzed with FCS version 3 software. IC50s were calculated by constructing a dose-response curve. Cell cycle was evaluated with PI staining: briefly, adherent cells and condrospheres were harvested in trypsin-EDTA, washed once with PBS, fixed in ice-cold 70\% ethanol, incubated with 50 $\mu \mathrm{g} / \mathrm{ml} \mathrm{PI} \mathrm{(Sigma)} \mathrm{plus} 1 \mathrm{mg} / \mathrm{ml} \mathrm{RNase}$ for $60 \mathrm{~min}$ at $4^{\circ} \mathrm{C}$ in the dark, and the data analyzed with Mod-Fit 2.0 cell cycle analysis software (BectonDickinson).

\section{Microarray analyses}

Microarray quality control reports generated by the Agilent Feature Extraction software were used to detect hybridization artifacts. Probe level raw intensity were processed using R/BioConductor [20,21] and Limma package.

Background correction was performed using "normexp" limma method and data normalization was carried out in two steps: loess normalization within-arrays to correct systematic dye-bias and quantile normalization between-arrays to detect systematic non-biological bias. Ratios representing the relative target mRNA intensities compared with control RNA probe signals were derived from normalized data.

Differentially expressed genes between conditions (chondrospheres 
Citation: Desiderio V, Paino F, Nebbioso A, Altucci L, Pirozzi G, et al. (2013) Molecular Profiling of Human Primary Chondrosarcoma-Derived Spheres Reveals Specific and Target Genes Involved in Multidrug Resistance and Metastasis. J Carcinogene Mutagene 5: 152. doi:10.4172/21572518.1000152

vs adherent cells) were identified using a paired Bayesian T-test $[22,23]$. For each p-value, the Benjamini-Hochberg procedure was used to calculate the False Discovery Rate (FDR) to avoid the problem of multiple testing. The selected gene lists were obtained using the following thresholds: FDR $<0.05$ and abs(ratio) $>2$. For a more stringent analysis, an FDR $<0.001$ and abs(ratio) $>3$ were also used.

Data have been deposited in NCBI's Gene Expression Omnibus (GEO) http://www.ncbi.nlm.nih.gov/geo/query/acc. cgi?acc=GSE47823) under the GEO series accession number GSE47823. The relative abundance of "Biological Process" ("Molecular function" and "Cellular Component") Gene Ontology terms in each of the selected lists was analyzed using the Database for Annotation, Visualization, and Integrated Discovery (DAVID) Functional Annotation Clustering tool [24].

\section{Validation of microarray data using quantitative TaqManH real-time PCR (qPCR)}

Condrospheres and adherent cells were harvested and total RNA extracted using the Pure Link RNA mini kit (Ambion, Life Technologies) according to the manufacturer's protocol. RNA was quantified by UVVis spectroscopy. Extracted RNA was treated with DNase I (Promega) and $1 \mu \mathrm{g}$ of total RNA was reverse-transcribed using SuperScript ${ }^{\circ}$ VILO $^{\text {TM }}$ master mix (Invitrogen).

qPCR analysis was performed using StepOne System (Applied Biosystem) and Sybr Select master mix (Applied Biosystem). The expression of GAPDH was used for normalization of gene expression values. Data were analyzed using the $2-{ }^{\Delta \Delta} \mathrm{Ct}$ method.

Primer pairs used are as follows: $C R O P$ (cisplatin resistance-associated overexpressed protein): fw 5'-AGGCGGAGCAGAAGTAGAGA-3'; rev 5'-TGACTTTCGATCCCGCTTT-3'; SOX2 (sex determining region Y-box 2): fw 5'-CGATGCCGACAAGAAACTT-3'; rev 5'CAATTCCTGCAAGCTCC-3'; p21 (cyclin-dependent kinase inhibitor 1A): fw 5'- AAGACCATGTGGACCTGTCACTGT-3', rev 5'-GAAGATCAGCCGGCGTTTG-3'; VIM (vimentin): fw 5'-CCTTGAACGCAAAGTGGAATC-3'; rev 5' GACATGCTGTTCCTGAATCTGAG-3'; B2M-microglobulin): fw 5'-TACATGTCTGATCCCACTTAAC-3'; rev 5'-GCTACTCCAAAGATTCAGGTT-3'; CD133 (PROM-1): fw 5'- TCTTGACCGACTGAGACCCAC-3'; rev 5'-ACTTGATGGATGCACCAAGCAC-3'; GAPDH (glyceraldehyde-3-phosphate dehydrogenase): fw 5'-TGGACTCCACGACGTACTCAG-3'; rev 5'-ACATGTTCCAATATGATTCCA-3'.

\section{Statistical analysis}

All the above-described experiments were performed in triplicate. Student t-test was used for statistical evaluation. The level of significance was set at $\mathrm{p}<0.05$

\section{Results}

\section{Cell culture and chondrospheres}

After five passages, isolated cells obtained from the chondosarcoma biopsy formed an adherent, homogeneous cell population, characterized mainly by small, chondrosarcoma-like cells (Figure 1A). When these primary culture cells were grown in condrosphere medium, sphere clusters were clearly observable already after 24 hours (Figure 1B). After 7 days of culture, chondrospheres were seeded in standard flasks and grown in DMEM with 10\% FBS. Polygonal-shaped cells migrated from the spheres within a few hours and adhered to the bottom of the flasks (Figure1C). Single cells from the spheres gave rise to secondary spheres that, in turn, were able to form tertiary spheres, as previously demonstrated by us [19]. Chondrospheres were passaged 20 times during the culture period.

\section{Chondrospheres contain cells expressing stemness markers}

The expression levels of stem cell markers connected to self-renewal/ stemness or differentiation capability were evaluated: these were OCT4, SOX2, and CD133 for stemness, and COL2 for differentiation. We found that OCT4-, SOX2-, or CD133-positive cells were significantly more numerous in sphere cultures than in adherent cultures. In contrast, COL2 was significantly less expressed in chondrospheres (Figure1D).

\section{Chondrospheres are resistant to cisplatin treatment}

The majority of the cells composing a tumor are killed off by the chemoradiotherapy normally used in standard treatments. However because of their heightened resistance to damage, CSCs may escape cell death. Therefore, we cultured chondrospheres and adherent cells with different concentrations of cisplatin and then calculated IC50 and analyzed apoptosis and cell cycle. The dose-response curves showed that IC50 for adherent cells was $70 \mu \mathrm{M}$, whereas for chondrospheres it was $>150 \mu \mathrm{M}$ (Figure 2A). Moreover, we found that for adherent cells, apoptosis was similar at $30 \mu \mathrm{M}$ and $50 \mu \mathrm{M}$, and then increased from 70 $\mu \mathrm{M}$ to $150 \mu \mathrm{M}$; in contrast, necrosis was similar at all concentrations (Figure 2B). In general, cell viability decreased in a dose-dependent manner for adherent cells. For chondrospheres, apoptosis was lower than for adherent cells and increased significantly only with exposure to $150 \mu \mathrm{M}$ cisplatin; necrosis, on the other hand, increased starting with exposure to $70 \mu \mathrm{M}$ cisplatin. The viability of chondrospheres started to decrease only at the highest concentrations of cisplatin (100$150 \mu \mathrm{M})$, remaining high at the lowest concentrations $(30-70 \mu \mathrm{M})$. Thus, compared with adherent cells, chondrospheres show a strong chemoresistance to cisplatin.

Regarding cell cycle, S-phase block was detectable in adherent cells: the number of cells in $\mathrm{G}_{0} \mathrm{G}_{1}$ and $\mathrm{G} 2 \mathrm{M}$ phases decreased especially at $100 \mu \mathrm{M}$ and $150 \mu \mathrm{M}$ (Figure $2 \mathrm{C}$ ). Here, sub- $\mathrm{G}_{0} \mathrm{G}_{1}$ picks were evident and attributable to apoptosis or necrosis due to cisplatin treatment. For chondrospheres, sub- $\mathrm{G}_{0} \mathrm{G}_{1}$ picks were similar at all concentrations tested and were lower than those of corresponding adherent cells. In addition, untreated chondrospheres had a greater number of cells in $G_{0} G_{1}$ than corresponding adherent cells, and they remained elevated along the whole series of cisplatin concentrations. Thus, high concentrations of cisplatin induced an S-phase block in adherent cells, but increased the number of chondrosphere cells in $\mathrm{G}_{0} \mathrm{G}_{1}$.

\section{Gene expression analysis}

Global gene regulation: Relative genome-wide changes in chondrosphere gene expression were determined by normalizing against the corresponding adherent cells as a control group. We found more than 20,000 differently expressed genes. To narrow down the frame of reference, a 2 -fold cut-off threshold was selected. This restricted our analysis to 1,405 genes: 629 (44.8\%) genes were up-regulated whereas $776(55.2 \%)$ genes were down-regulated in chondrospheres vs adherent cells. In particular, 540 genes were up-regulated with a fold change between 1.0 and $1.99,72$ genes with fold change ranging from 2.0 to 2.9, and 13 genes with fold change ranging from 3.0 to 3.9. Moreover, 676 genes were down-regulated with a fold change between -1.0 and $-1.9,93$ genes with fold change ranging from -2.0 to -2.9 , and 7 genes with fold change from -3.1 to -3.7 . As a more stringent analysis, an $\mathrm{FDR}<0.001$ was also selected. In this setting, the number of differentially expressed probes decreased to 14,067 . In addition, with a 3 -fold cut-off 
Citation: Desiderio V, Paino F, Nebbioso A, Altucci L, Pirozzi G, et al. (2013) Molecular Profiling of Human Primary Chondrosarcoma-Derived Spheres Reveals Specific and Target Genes Involved in Multidrug Resistance and Metastasis. J Carcinogene Mutagene 5: 152. doi:10.4172/21572518.1000152

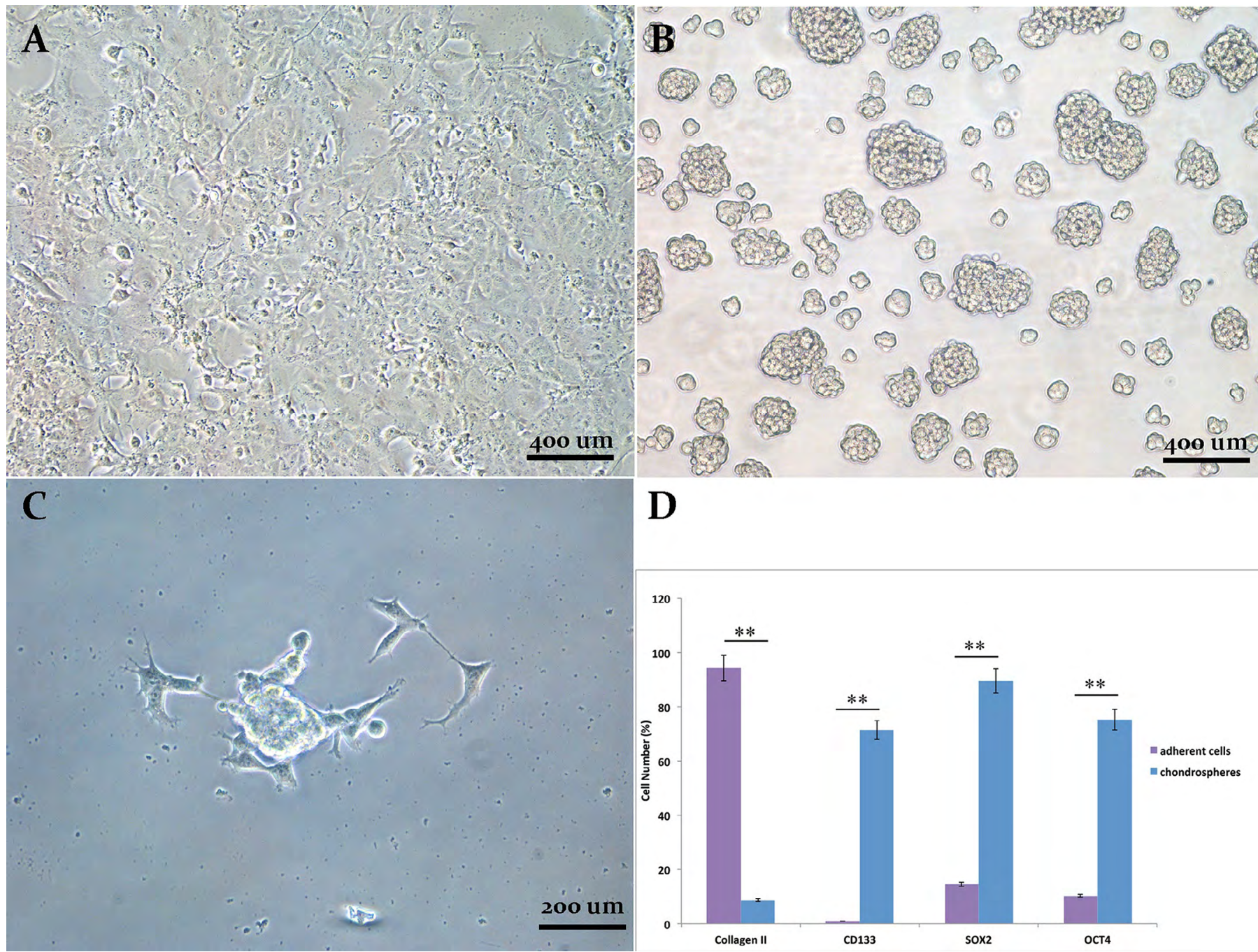

Figure 1: Chondrosphere formation and cytometric analyses. (A) Adherent, homogeneous chondrosarcoma cells. Scale bar $=400 \mu \mathrm{m}$; $(\mathrm{B})$ Chondrosphere clusters were clearly observable already after 24 hours of culture in condrosphere medium. Scale bar $=400 \mu \mathrm{m}$; (C) Cells migrated from the spheres within a few hours of culture in standard culture-ware and adhered to the bottom of the flasks, assuming a polygonal shape. Scale bar $=200 \mu \mathrm{m}$; (D) Number of cells positive for OCT4, SOX2, CD133, and collagen type II in chondrospheres and adherent cells. All error bars represent $95 \%$ confidence intervals; ${ }^{* *} p<0.005$ compared with adherent cells.

threshold, the number of up-regulated and down-regulated probes was 251 and 302 , respectively.

Using Gene Ontology (GO) classification, the identified probe sets were subdivided according to their function into cell motility, regulation of apoptosis, cell cycle regulation, immune response regulation, and stemness (Figure 3A)

Stemness: For stemness, 43 genes were analyzed. Of these, the differences for 26 genes were statistically significant: 18 (69.2\%) genes were up-regulated, including NANOG, POU5F1, LIF, NOG, and FZD7 [25-28], whereas 8 (30.8\%) genes were down-regulated in chondrospheres vs adherent cells (Supplementary Table 1 and Figure 3B). Therefore, $69.2 \%$ of stemness genes were up-regulated while $30.8 \%$ were down-regulated. In particular, POU5F1, LIF, and NANOG are involved in maintaining pluripotency $[25,26]$. Up-regulation of PROM1 [29] in chondrospheres - which was validated by qPCR (Figure 4) reinforced the hypothesis that they were formed from stem cells.
Multidrug resistance: Interestingly, all tested genes involved in multidrug resistance were up-regulated in chondrospheres vs adherent cells (Figure $3 \mathrm{~B}$ ) with an $\mathrm{FDR}<0.05$ and fold change $=2$. $A B C C 2$ and TAP1 belong to the superfamily of ATP-binding cassette $(\mathrm{ABC})$ transporters. $\mathrm{ABC}$ proteins transport various molecules across extra- and intra-cellular membranes. TAP1 encodes for a protein belonging to the MDR/TAP subfamily and involved in the pumping of degraded cytosolic peptides across the endoplasmic reticulum into the membrane-bound compartment where class I molecules assemble. $A B C C 2$ and TAP1 were up-regulated with fold changes of 1.49 and 1.65 , respectively. Of note, CROP 1 - encoding a protein associated with cisplatin resistance - was up-regulated in chondrospheres (Figure 3B). Up-regulation of CROP1 was confirmed by qPCR (Figure 4). With an FDR $<0.001$ and fold change $=3$, only TAP1 was found up-regulated (Supplementary Table 2).

Cell death and regulation of the cell cycle: For apoptosis and cell cycle regulation, 258 genes were analyzed: a total of 102 cell death genes 
Citation: Desiderio V, Paino F, Nebbioso A, Altucci L, Pirozzi G, et al. (2013) Molecular Profiling of Human Primary Chondrosarcoma-Derived Spheres Reveals Specific and Target Genes Involved in Multidrug Resistance and Metastasis. J Carcinogene Mutagene 5: 152. doi:10.4172/21572518.1000152

A

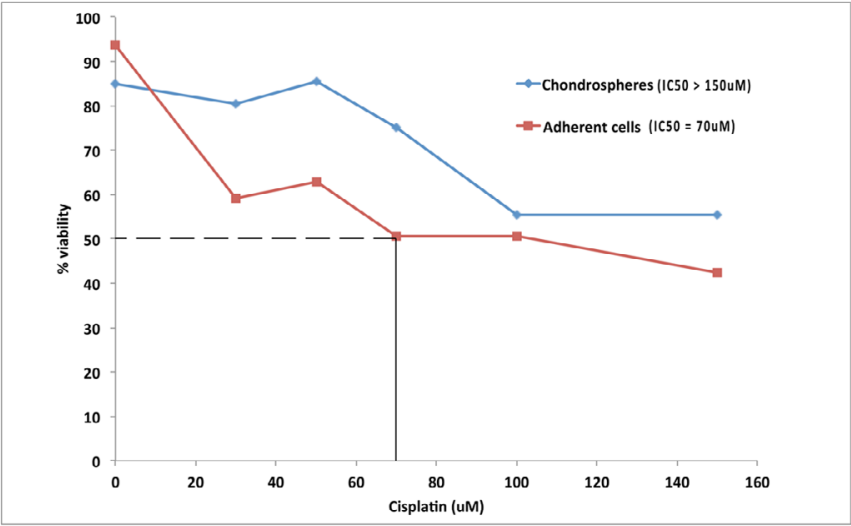

B

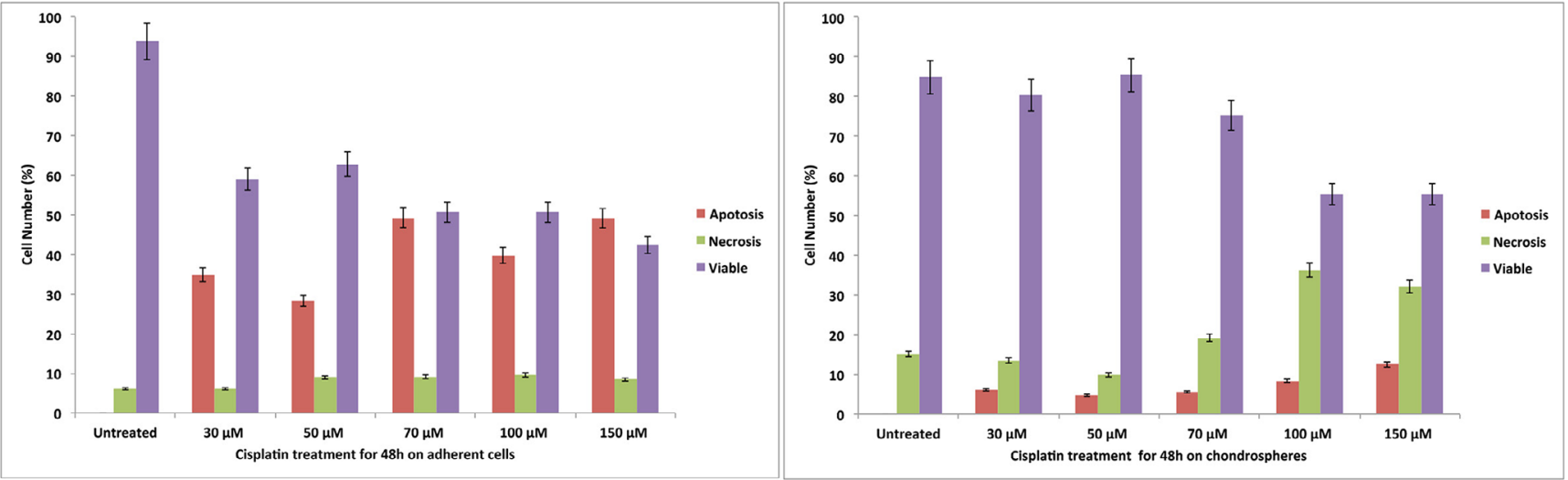

C
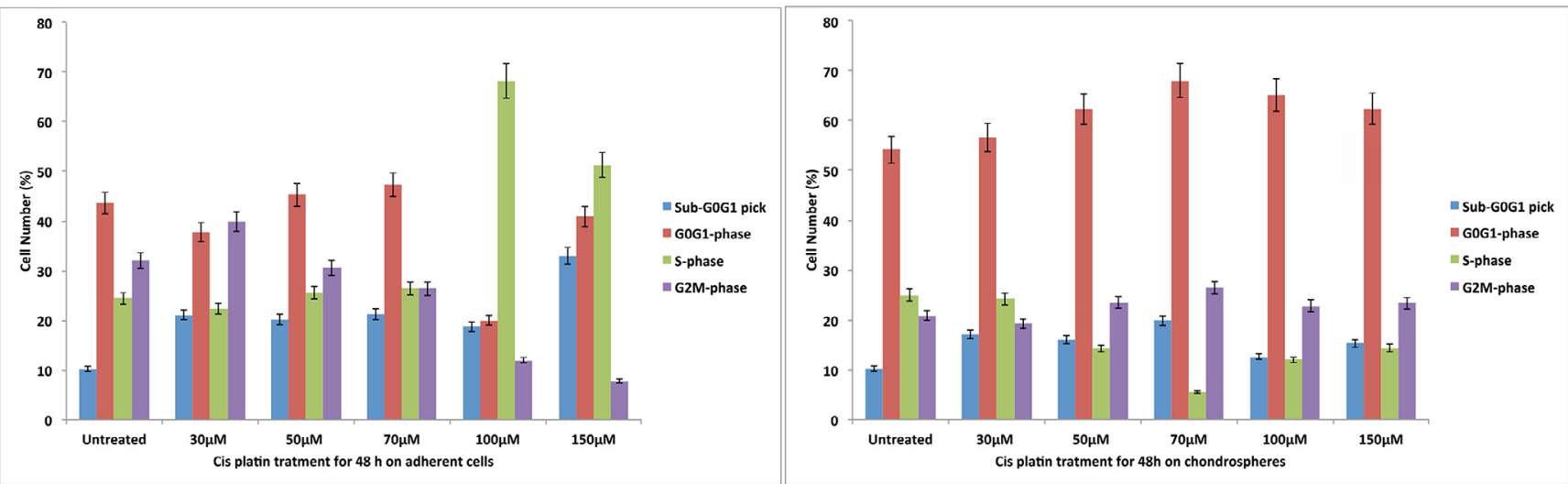

Figure 2: IC50 evaluation and apoptosis and cell cycle analyses. (A) Dose-response curves revealed IC50 is $70 \mu \mathrm{M}$ for adherent cells and it is $>150 \mu \mathrm{M}$ for chondrospheres; (B) chondrospheres showed profound chemoresistance to cisplatin compared with the adherent cells; (C) Cisplatin treatment induced S-phase block in adherent cells and an increase in G0G1-phase chondrosphere cells only at high concentrations.

and 156 cell cycle genes were differentially expressed with an $\mathrm{FDR}<0.05$ and fold change $=2$ (Figure 3B). In particular, 57 genes were involved in apoptosis. Interestingly, all genes tested were implicated in negative regulation of programmed cell death. Of these, 35 (61.4\%) were upregulated and $22(38.6 \%)$ were down-regulated, demonstrating that antiapoptotic mechanisms were activated in chondrospheres, as reported in Figure 3B (apoptosis panel). 37 genes were found involved in apoptosis with an FDR $<0.001$ and fold change $=3$ : $64.8 \%$ were up-regulated and $35.2 \%$ were down-regulated, as reported in Supplementary Table 3. On the whole, cell death genes were repressed in chondrospheres.

For cell cycle, 156 gene differences were statistically significant with an FDR $<0.05$ and fold change $=2$. Of these, 31 genes were up-regulated
(19.8\%) and 125 genes were down-regulated (80.2\%) (Figure 3B). In particular, most of the down-regulated genes were involved in cell cycle promotion. Aurora kinase B was down-regulated, negatively mediating G2-to-M phase transition and cytokinesis. In addition, $C D C 2, C D C 20$, $C D C 25 B$, and $C D C 45 L$ were down-regulated, inhibiting both G2-to-M phase and G1-to-S transition and mitosis. CDC6, which is expressed in all proliferating cells but not in quiescent or differentiated cells, was also down-regulated, confirming again the quiescence of chondrosphere cells. Other down-regulated genes included $C D C A 2, C D C A 3, C D C A 5$, CDCA7, and CDCA8, which are implicated in regulating chromatin structure during mitosis. Up-regulation of p21 was confirmed by qPCR (Figure 4). 
Citation: Desiderio V, Paino F, Nebbioso A, Altucci L, Pirozzi G, et al. (2013) Molecular Profiling of Human Primary Chondrosarcoma-Derived Spheres Reveals Specific and Target Genes Involved in Multidrug Resistance and Metastasis. J Carcinogene Mutagene 5: 152. doi:10.4172/21572518.1000152

A
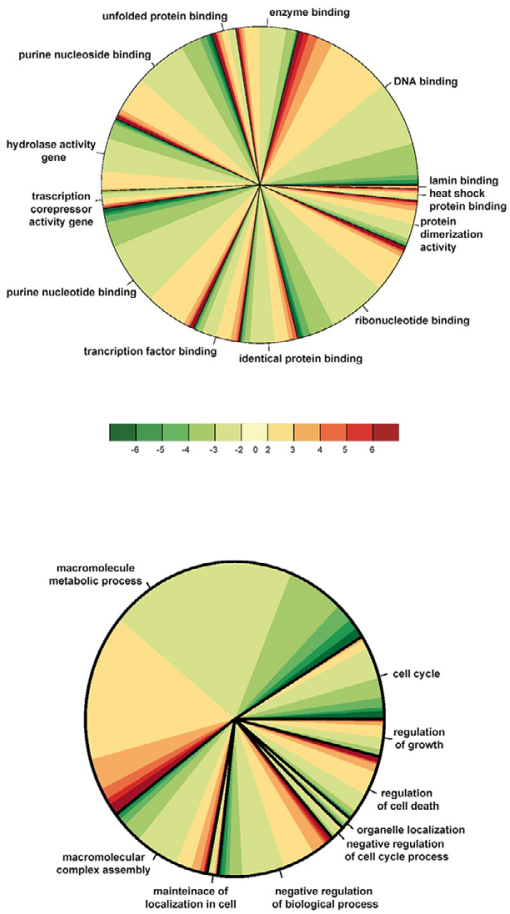

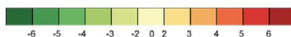

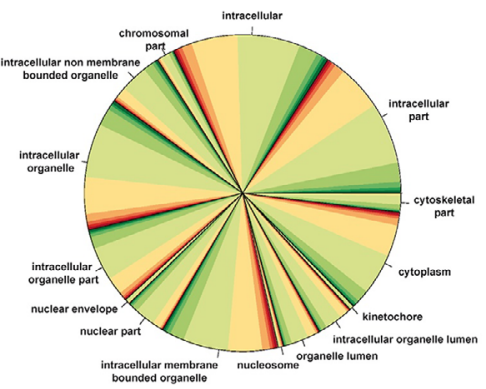

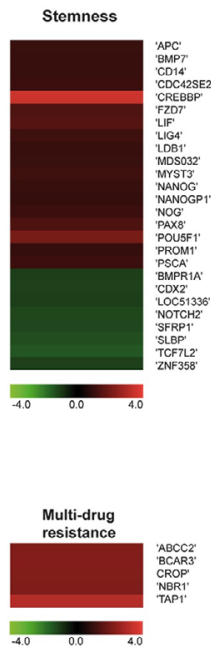

Apoptosis

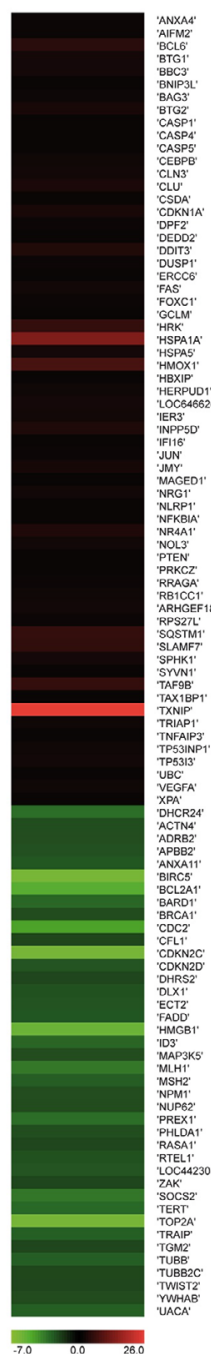

Cell cycle
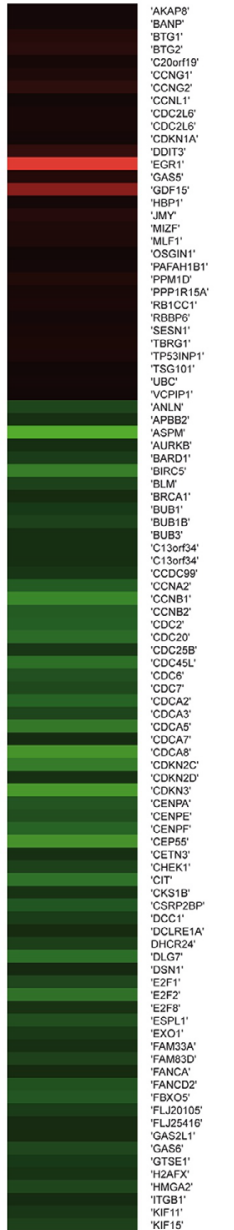

Immuno-respons
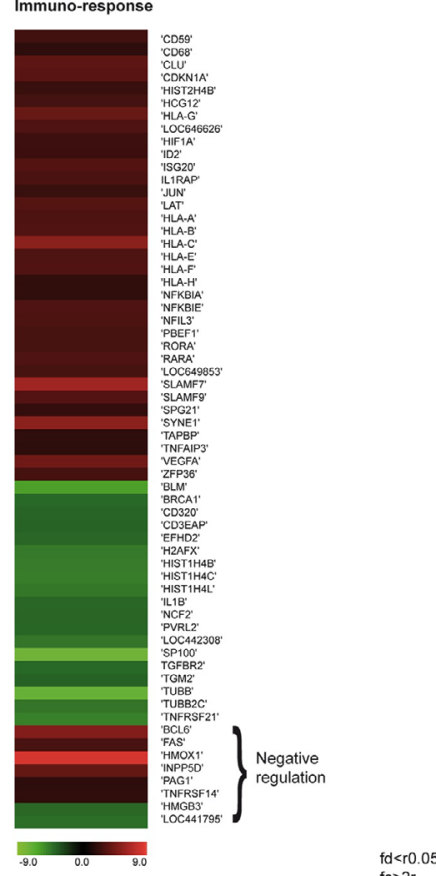

Figure 3: Gene ontology and expression data. A) Gene Ontology (GO) pie chart of the total list of 1,405 genes classified according to molecular function (MF, upper), biological function (BP, middle), and cellular component (CC, lower); B) Heat-map (obtained using the MEV platform) showing the expression fold change of genes (with two fold or more) divided into 9 clusters according to GO terms. Red/green indicate an increase/decrease in gene expression. 


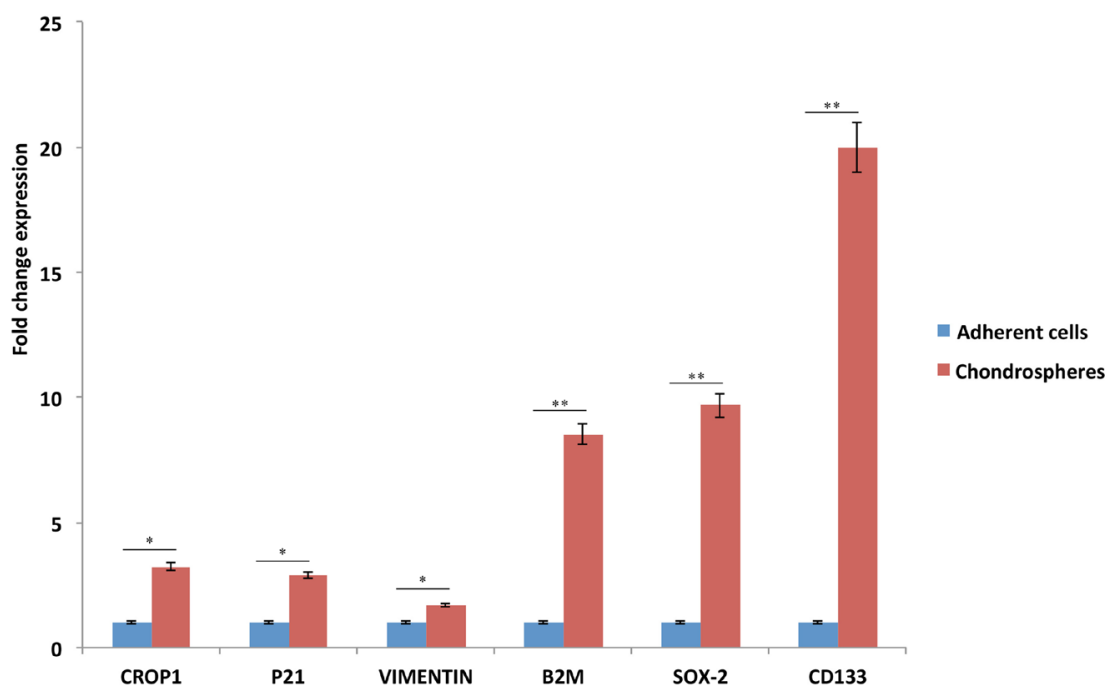

Figure 4: Validation of microarray data using quantitative TaqManH real-time PCR. Fold change expression of $C R O P 1, p 21, V I M, B 2 M, S O X 2$, and $C D 133$ for condrospheres versus adherent cells. Error bars represent $95 \%$ confidence intervals; ${ }^{* *}, p<0.005$ and ${ }^{* * *}, p<0.001$ compared with adherent cells.

With an FDR $<0.001$ and fold change $=3,86$ gene differences were statistically significant. Of these, $12.8 \%$ were up-regulated and $87.2 \%$ genes were down-regulated. Also in this case, $C D C 2, C D C 20$, CDC45L,CDC6, CDCA2, CDCA3, CDCA5, CDCA7, and CDCA8 resulted to be down-regulated (Supplementary Table 4).

Angiogenesis, migration, motility, and invasion: For angiogenesis, 7 genes were significantly differentially expressed with an FDR $<0.05$ and fold change $=2$ (Figure $3 \mathrm{~B}$ ): $5(71.4 \%)$ were up-regulated and $2(28.6 \%)$ were down-regulated in chondrospheres vs adherent cells. VEGFA, VASN, ANG, VWCE, and GMFG were up-regulated with a fold change $>1$.

With an FDR $<0.001$ and fold change $=3,4$ gene differences were statistically significant. Of these, $75 \%$ were up-regulated and $25 \%$ genes were down-regulated. In this case, up-regulated genes were VEGFA, $A N G$, and VWCE (Supplementary Table 5).

For metastasis, motility, and invasion, 68 genes were found with an FDR $<0.05$ and fold change $=2: 36(53 \%)$ were up-regulated and 32 $(47 \%)$ were down-regulated (Figure $3 \mathrm{~B}$ ). With an FDR $<0.001$ and fold change $=3,29$ genes were significantly expressed, $48.3 \%$ of which were up-regulated and $51.7 \%$ of which were down-regulated (Supplementary Table 6). Overall, metastasis and angiogenesis genes were regulated positively. Vimentin was validated by qPCR (Figure 4).

Immune response and cell growth: For immune response modulation, 64 genes were found with an $\mathrm{FDR}<0.05$ and fold change $=2$ (Figure 3B): $42(65.6 \%)$ genes were up-regulated with a fold change ranging from 1.04 to 2.4 , and $22(34.4 \%)$ genes were down-regulated with a fold change between -1.0 and -1.4. In particular, genes codifying for major histocompatibility complex (HLA) class I types A, B, C, E, $\mathrm{F}, \mathrm{G}$, and $\mathrm{H}$ were up-regulated. Overall, genes involved in the innate immune response and in positive regulation of the immune response showed a balance between up- and down-regulation. For genes involved in negative regulation of the immune response, 6 genes were up-regulated and 2 down-regulated (Figure 3B).

With an FDR $<0.001$ and fold change $=3,24$ genes resulted to be differentially expressed. In particular, $87.5 \%$ were up-regulated and $12.5 \%$ down-regulated. Genes codifying for HLA class I proteins were up-regulated (Supplementary Table 7). Three genes involved in negative regulation were up-regulated (Supplementary Table 8).

For cell growth regulation, 43 genes were found with an FDR $<0.05$ and fold change $=2: 22(51.1 \%)$ genes were up-regulated and $21(48.9 \%)$ were down-regulated (Figure $3 \mathrm{~B}$ ).

With an FDR $<0.001$ and fold change $=3,21$ genes resulted to be differentially expressed. In particular, $52.4 \%$ were up-regulated, while $47.6 \%$ were down-regulated (Supplementary Table 9). Taking into consideration all the different functions and different conditions, cell growth was negatively regulated. HLA class I was validated by qPCR (Figure 4).

\section{Discussion}

Cancers are composed of heterogeneous cell populations and are defined on the basis of three main characteristics: 1) a selective tumorigenic capacity; 2) self-renewal and differentiation, i.e., the ability to sustain growth of heterogeneous cancer tissues; and 3) expression of specific surface markers, allowing reproducible selection [30]. CSCs can be selected from tumors through their ability to grow as spheres [31]. Weiss and colleagues [32] showed in neurospheres that between $4 \%$ and $20 \%$ of cells were stem cells, while the others were progenitor cells in different phases of differentiation [32,33]. Stem/progenitor cells were also enriched from mammary cell populations on the basis of their ability to grow as spheres (mammospheres) [34]. Subsequently, sphere culture techniques have been applied to cell populations from a variety of cancers, such as brain cancers, breast cancers, lung cancers, and melanomas, with successful enrichment of cells with CSC features [35-38].

The present study confirms and expands the recent observations showing that chondrosarcomas are a source of cancer stem cells $[19,39]$ and demonstrates that chondrospheres may constitute an excellent model for the study of tumoral heterogeneity and multidrug resistance. The molecular pathways controlling genesis and growth of chondrosarcoma have not been defined until now. By analyzing the global pattern of gene expression, we define a signature that is distinctive for sphere vs adherent cell cultures of human chondrosarcoma. 
Citation: Desiderio V, Paino F, Nebbioso A, Altucci L, Pirozzi G, et al. (2013) Molecular Profiling of Human Primary Chondrosarcoma-Derived Spheres Reveals Specific and Target Genes Involved in Multidrug Resistance and Metastasis. J Carcinogene Mutagene 5: 152. doi:10.4172/21572518.1000152

In fact, our microarray analysis revealed that adherent cells and chondrospheres differ substantially in their pattern of gene expression. First, we studied the expression of a well-defined set of known stemness markers, such as OCT4, NANOG, and SOX2 [25], by FACS, qRT$P C R$, and microarray and found, as expected, that these genes are upregulated in chondrospheres. Also, genes such as LIF, NOG, and FZD7 resulted to be up-regulated. These genes contribute to and support selfrenewal signaling of human embryonic stem cells [26-28]. Secondly, we compared the global pattern of gene expression of chondrospheres vs adherent cells. We used two analysis settings: 1 ) FDR $<0.05$ with fold change $=2$ and 2) $\mathrm{FDR}<0.001$ with fold change $=3$. With the first, we identified a set of 629 up-regulated and 776 down-regulated genes, whereas with second, a set of 251 up-regulated and 302 down-regulated genes was identified in condrospheres.

Three main results are noteworthy. First, multidrug resistance markers - CROP1 above all - are differentially up-regulated in chondrospheres, a finding that is associated with increased cisplatin resistance of sphere cells compared with their adherent counterparts. CROP1 encodes for the cisplatin resistance-associated over-expressed protein (CROP). The N-terminal half of CROP contains cysteine/ histidine motifs and leucine zipper-like repeats, whereas the $\mathrm{C}$-terminal half is rich in arginine and glutamate residues (RE domain) as well as arginine and serine residues (RS domain). This protein localizes with a speckled pattern to the nucleus, and could be involved in the formation of the splicesome via the RE and RS domains. Two alternatively spliced transcript variants encoding the same protein have been found for this gene, conferring cisplatin resistance [40]. Although CROP1 resulted to be up-regulated with an FDR $<0.05$ and fold change $=2$, we consider this finding very important. In fact, because CROP1 is involved in splicing processes, even a small variation may lead to a remarkable biological effect. Thus, CROP1 probably plays a key role in addition to other multidrug resistance factors - such as ABCC1, BCAR3, TAP1, and NBR1 - in the notorious resistance to chemoradiotherapy of chondrosarcoma $[1,41]$.

Although the expression of $\mathrm{ABC}$ transporters as well as CROP could render chondrospheres resistant to drugs, it is not the sole determinant of resistance, as both quiescence of the cells and the reluctance to undergo apoptosis could be equally or even more important. Generally regarded as quiescent and non-dividing [42], stem cells would be expected to be inherently refractory to drugs that target either the cell cycle or rapidly dividing cells. In line with this, we found that there was an inhibition of apoptosis- and cell cycle-related genes in chondrospheres, confirming the hypothesis that quiescence and negative regulation of cell cycle and apoptosis, in addition to overexpression of multidrug resistance genes, may be involved in the resistance to treatment that is typical of chondrosarcoma.

The second prominent result concerns two of the most important traits of cancer: angiogenesis and the development of metastases. Chondrospheres had differentially up-regulated VEGFA, VWCE, ANG, and MALAT1, factors involved in tumor angiogenesis and metastasis. Tumor vascularity and the factors that stimulate angiogenesis have been shown to be correlated with biologic aggressiveness, high pathological grade, and poor survival [43]. In fact, once a tumor grows beyond several millimeters in diameter, angiogenesis must occur to support further growth. Moreover, the first step in metastasis is for the tumor cell to gain access to the circulation. Neo-formed vessels increase permeability and allow tumor cells to egress into the circulation and initiate metastasis. In particular, VEGF-A is a basic, homodimeric glycoprotein of approximately 45,000 Da that is expressed in four isoforms via alternative exon splicing of the gene [44]. It is the most commonly studied angiogenic cytokine, the primary inducer of angiogenesis in multiple types of carcinomas, and has been strongly correlated with biologic aggressiveness, metastasis, and prognosis [45]. On the other hand, MALAT1 regulates both the expression of metastasis-associated genes and cell motility via transcriptional and/ or post-transcriptional regulation of motility-related genes [46-49]. It is up-regulated in several solid tumors and is a highly abundant nucleusrestricted RNA that localizes to nuclear speckles, a sub-nuclear domain suggested to coordinate RNA polymerase II transcription, pre-mRNA splicing, and mRNA export. MALAT1 interacts with several premRNA splicing factors, including serine arginine dipeptide-containing SR family splicing factors, thereby influencing alternative splicing of pre-mRNAs [50,51]. Through such a mechanism, cells could alter the local concentration of a particular splicing factor upon a specific external signal or during specific stages of the cell cycle [51].

Finally, a result that deserves to be discussed regards immune response modulation. Overall, we found a balance between up- and down-regulated genes in chondrosphere cells; however, negative modulation of the immune response seemed to be favored. Notably, HLA class I genes appeared to be up-regulated. Little is known concerning the antigen processing and presentation machinery in CSCs and normal stem cells, let alone peptide-HLA complexes unique to stem cells that may serve an immunological target. Usually, loss of HLA class I antigens appears to be a significant mechanism by which tumor cells escape specific immune attack and cause problems in the design of antitumor immunotherapy. The loss of HLA class I antigens on tumor cells has been reported in several human tumors, and the loss of HLA class I molecules has been discussed in the context of tumor aggressiveness, such as differentiation of histology, invasiveness, and metastatic potential [52]. Our result seems to be in conflict with these data. Despite this, Aptsiauri et al. [53] showed that if apparent tumor cells expressed HLA class I, various types of HLA class I alterations were found in malignancies and in the molecular mechanisms that underlie these defects. In this context, the HLA class I molecules preserved in breast cancers may exhibit altered expression and dysfunction as antigen presentation molecules [54]. There are several mechanisms proposed to explain abnormal HLA class I phenotypes: 1) impaired transcriptional activity of genes codifying for HLA class I; 2) deregulation of antigen-processing machinery components responsible for functional HLA class I expression, such as $\beta-2$ microglobulin and transporters associated with antigen processing; and 3) degradation of HLA class I proteins by proteasomes [55].

In fact, HLA-G, which is considered to be one of the major factors in the immune mechanism preventing the rejection of the semi-allogenic fetus in the mother, suppresses the proliferation of $\mathrm{CD} 4^{+} \mathrm{T}$ lymphocytes in vitro, induces apoptosis in activated $\mathrm{CD} 8^{+} \mathrm{T}$ lymphocytes, and inhibits the cytotoxicity of natural killer cells [56]. Therefore, although there was an up-regulation of HLA genes involved in the immune response, in chondrosphere cells, other mechanisms must be considered.

In conclusion, chondrospheres express stemness-, invasion-, multidrug resistance- and metastasis-related genes and are highly resistant to cisplatin treatment. Our study highlights that either CROP1 and TAP1 genes, involved in multidrug resistance, or VEGFA and $M A L A T 1$ genes, involved in angiogenesis and metastasis, are strongly up-regulated, and could be considered potential therapeutic targets for the treatment of chondrosarcoma. In this context, effects of targeting VEGFA and MALAT1 genes, have been described in in vivo models $[57,58]$. Phase I studies have reported a response of chondrosarcomas 
Citation: Desiderio V, Paino F, Nebbioso A, Altucci L, Pirozzi G, et al. (2013) Molecular Profiling of Human Primary Chondrosarcoma-Derived Spheres Reveals Specific and Target Genes Involved in Multidrug Resistance and Metastasis. J Carcinogene Mutagene 5: 152. doi:10.4172/21572518.1000152

to new target agents such as antisense VEGF [59]. Therefore, because the treatment of chondrosarcoma is mainly based on surgery, it appears clear that a better understanding of chondrosarcoma biology could open new, non-surgical therapeutic options.

\section{Disclosure}

The authors have nothing to disclose.

\section{Conflict of interest}

The authors declare no conflict of interest.

\section{Acknowledgment}

Financial support: European Grant PON01_02834 Prometheus to GP

\section{References}

1. Gelderblom H, Hogendoorn PC, Dijkstra SD, van Rijswijk CS, Krol AD, et al. (2008) The clinical approach towards chondrosarcoma. Oncologist 13: 320329 .

2. Healey JH, Lane JM (1986) Chondrosarcoma. Clin Orthop Relat Res : 119-129.

3. Morioka H, Weissbach L, Vogel T, Nielsen GP, Faircloth GT, et al. (2003) Antiangiogenesis treatment combined with chemotherapy produces chondrosarcoma necrosis. Clin Cancer Res 9: 1211-1217.

4. DeLaney TF, Liebsch NJ, Pedlow FX, Adams J, Dean S, et al. (2009) Phase II study of high-dose photon/proton radiotherapy in the management of spine sarcomas. Int J Radiat Oncol Biol Phys 74: 732-739.

5. Schrage YM, Machado I, Meijer D, Briaire-de Bruijn I, van den Akker BE, et al. (2010) COX-2 expression in chondrosarcoma: a role for celecoxib treatment? Eur J Cancer 46: 616-624.

6. Björnsson J, McLeod RA, Unni KK, Ilstrup DM, Pritchard DJ (1998) Primary chondrosarcoma of long bones and limb girdles. Cancer 83: 2105-2119.

7. Rizzo M, Ghert MA, Harrelson JM, Scully SP (2001) Chondrosarcoma of bone: analysis of 108 cases and evaluation for predictors of outcome. Clin Orthop Relat Res : 224-233.

8. Fiorenza F, Abudu A, Grimer RJ, Carter SR, Tillman RM, et al. (2002) Risk factors for survival and local control in chondrosarcoma of bone. J Bone Joint Surg $\mathrm{Br}$ 84: 93-99.

9. Pritchard DJ, Lunke RJ, Taylor WF, Dahlin DC, Medley BE (1980) Chondrosarcoma: a clinicopathologic and statistical analysis. Cancer 45: 149157.

10. Lee FY, Mankin HJ, Fondren G, Gebhardt MC, Springfield DS, et al. (1999) Chondrosarcoma of bone: an assessment of outcome. J Bone Joint Surg Am 81: 326-338.

11. Tirino V, Desiderio V, Paino F, De Rosa A, Papaccio F, et al. (2013) Cancer stem cells in solid tumors: an overview and new approaches for their isolation and characterization. FASEB J 27: 13-24.

12. Reynolds BA, Weiss S (1992) Generation of neurons and astrocytes from isolated cells of the adult mammalian central nervous system. Science 255 : 1707-1710.

13. Gibbs CP, Kukekov VG, Reith JD, Tchigrinova O, Suslov ON, et al. (2005) Stem-like cells in bone sarcomas: implications for tumorigenesis. Neoplasia 7: 967-976.

14. Fan $X$, Ouyang $\mathrm{N}$, Teng $\mathrm{H}$, Yao $\mathrm{H}$ (2011) Isolation and characterization of spheroid cells from the HT29 colon cancer cell line. Int J Colorectal Dis 26: 1279-1285.

15. Lobo NA, Shimono Y, Qian D, Clarke MF (2007) The biology of cancer stem cells. Annu Rev Cell Dev Biol 23: 675-699.

16. Jung $P$, Sato T, Merlos-Suárez A, Barriga FM, Iglesias M, et al. (2011) Isolation and in vitro expansion of human colonic stem cells. Nat Med 17: 1225-1227.

17. Hansford LM, McKee AE, Zhang L, George RE, Gerstle JT, et al. (2007) Neuroblastoma cells isolated from bone marrow metastases contain a naturally enriched tumor-initiating cell. Cancer Res 67: 11234-11243.

18. Tirino V, Desiderio V, d'Aquino R, De Francesco F, Pirozzi G, et al. (2008) Detection and characterization of CD133+ cancer stem cells in human solid tumours. PLoS One 3: e3469.
19. Tirino V, Desiderio V, Paino F, De Rosa A, Papaccio F, et al. (2011) Human primary bone sarcomas contain CD133+ cancer stem cells displaying high tumorigenicity in vivo. FASEB J 25: 2022-2030.

20. Gentleman RC, Carey VJ, Bates DM, Bolstad B, Dettling M, et al. (2004) Bioconductor: open software development for computational biology and bioinformatics. Genome Biol 5: R80.

21. Smyth GK (2005) Limma: linear models for microarray data, in: R. Gentleman V. Carey, S. Dudoit, R. Irizarry, W. Bioinformatics and Computational Biology Solutions using R and Bioconductor. Huber editors. New York: Springer, pp. 397-420.

22. Baldi P, Long AD (2001) A Bayesian Framework for the Analysis of Microarray Expression Data: Regularized t-Test and Statistical Inferences of Gene Changes. Bioinformatics. 17: 509-519.

23. Benjamini Y, Hochberg $Y$ (1995) Controlling the False Discovery Rate: A Practical and Powerful Approach to Multiple Testing. Journal of the Royal Statistical Society. Series B Methodological. 57: 289-300.

24. Huang da W, Sherman BT, Lempicki RA (2009) Systematic and integrative analysis of large gene lists using DAVID bioinformatics resources. Nat Protoc 4: 44-57.

25. Boyer LA, Lee TI, Cole MF, Johnstone SE, Levine SS, et al. (2005) Core transcriptional regulatory circuitry in human embryonic stem cells. Cell 122 947-956.

26. Hsieh YC, Intawicha $P$, Lee KH, Chiu YT, Lo NW, et al. (2011) LIF and FGF cooperatively support stemness of rabbit embryonic stem cells derived from parthenogenetically activated embryos. Cell Reprogram 13: 241-255.

27. Krause C, Guzman A, Knaus P (2011) Noggin. Int J Biochem Cell Biol 43: 478 481

28. Melchior K, Weiss J, Zaehres H, Kim YM, Lutzko C, et al. (2008) The WNT receptor FZD7 contributes to self-renewal signaling of human embryonic stem cells. Biol Chem 389: 897-903.

29. Li Z (2013) CD133: a stem cell biomarker and beyond. Exp Hematol Oncol 2 17.

30. Reya T, Morrison SJ, Clarke MF, Weissman IL (2001) Stem cells, cancer, and cancer stem cells. Nature 414: 105-111.

31. Tirino V, Desiderio V, Paino F, Papaccio G, De Rosa M (2012) Methods for cancer stem cell detection and isolation. Methods Mol Biol 879: 513-529.

32. Weiss S, Dunne C, Hewson J, Wohl C, Wheatley M, et al. (1996) Multipotent CNS stem cells are present in the adult mammalian spinal cord and ventricular neuroaxis. J Neurosci 16: 7599-7609.

33. Reynolds BA, Weiss S (1996) Clonal and population analyses demonstrate that an EGF-responsive mammalian embryonic CNS precursor is a stem cell. Dev Biol 175: 1-13.

34. Dontu G, Abdallah WM, Foley JM, Jackson KW, Clarke MF, et al. (2003) In vitro propagation and transcriptional profiling of human mammary stem/progenitor cells. Genes Dev 17: 1253-1270.

35. Singh SK, Clarke ID, Terasaki M, Bonn VE, Hawkins C, et al. (2003) Identification of a cancer stem cell in human brain tumors. Cancer Res. 63: 5821-5828.

36. Ponti D, Costa A, Zaffaroni N, Pratesi G, Petrangolini G, et al. (2005) Isolation and in vitro propagation of tumorigenic breast cancer cells with stem/progenitor cell properties. Cancer Res 65: 5506-5511.

37. Fang D, Nguyen TK, Leishear K, Finko R, Kulp AN, et al. (2005) A tumorigenic subpopulation with stem cell properties in melanomas. Cancer Res 65: 93289337.

38. Tirino V, Camerlingo R, Franco R, Malanga D, La Rocca A, et al. (2009) The role of CD133 in the identification and characterisation of tumour-initiating cells in non-small-cell lung cancer. Eur J Cardiothorac Surg 36: 446-453.

39. Boeuf S, Kunz P, Hennig T, Lehner B, Hogendoorn P, et al. (2008) A chondrogenic gene expression signature in mesenchymal stem cells is a classifier of conventional central chondrosarcoma. J Pathol 216: 158-166.

40. Umehara H, Nishii Y, Morishima M, Kakehi Y, Kioka N, et al. (2003) Effect of cisplatin treatment on speckled distribution of a serine/arginine-rich nuclear protein CROP/Luc7A. Biochem Biophys Res Commun 301: 324-329.

41. Moussavi-Harami F, Mollano A, Martin JA, Ayoob A, Domann FE, et al. (2006) Intrinsic radiation resistance in human chondrosarcoma cells. Biochem Biophys Res Commun 346: 379-385. 
Citation: Desiderio V, Paino F, Nebbioso A, Altucci L, Pirozzi G, et al. (2013) Molecular Profiling of Human Primary Chondrosarcoma-Derived Spheres Reveals Specific and Target Genes Involved in Multidrug Resistance and Metastasis. J Carcinogene Mutagene 5: 152. doi:10.4172/21572518.1000152

42. Moore N, Lyle S (2011) Quiescent, slow-cycling stem cell populations in cancer: a review of the evidence and discussion of significance. J Oncol 2011

43. Kirsch M, Schackert G, Black PM (2004) Metastasis and angiogenesis. Cancer Treat Res 117: 285-304.

44. Shinkaruk S, Bayle M, Laïn G, Déléris G (2003) Vascular endothelial cell growth factor (VEGF), an emerging target for cancer chemotherapy. Curr Med Chem Anticancer Agents 3: 95-117.

45. Carmeliet $P$ (2005) VEGF as a key mediator of angiogenesis in cancer. Oncology 69 Suppl 3: 4-10.

46. Gutschner T, Hämmerle M, Eissmann M, Hsu J, Kim Y, et al. (2013) The noncoding RNA MALAT1 is a critical regulator of the metastasis phenotype of lung cancer cells. Cancer Res 73: 1180-1189.

47. Zong X, Tripathi V, Prasanth KV (2011) RNA splicing control: yet another gene regulatory role for long nuclear noncoding RNAs. RNA Biol 8: 968-977.

48. Gibb EA, Vucic EA, Enfield KS, Stewart GL, Lonergan KM, et al. (2011) Human cancer long non-coding RNA transcriptomes. PLoS One 6: e25915.

49. Ji P, Diederichs S, Wang W, Böing S, Metzger R, et al. (2003) MALAT-1, a nove noncoding RNA, and thymosin beta4 predict metastasis and survival in earlystage non-small cell lung cancer. Oncogene 22: 8031-8041.

50. Tollervey JR, Curk T, Rogelj B, Briese M, Cereda M, et al. (2011) Characterizing the RNA targets and position-dependent splicing regulation by TDP-43. Nat Neurosci 14: 452-458.

51. Tripathi V, Ellis JD, Shen Z, Song DY, Pan Q, et al. (2010) The nuclear-retained noncoding RNA MALAT1 regulates alternative splicing by modulating SR splicing factor phosphorylation. Mol Cell 39: 925-938.
52. Bukur J, Jasinski S, Seliger B (2012) The role of classical and non-classical HLA class I antigens in human tumors. Semin Cancer Biol 22: 350-358.

53. Aptsiauri N, Cabrera T, Mendez R, Garcia-Lora A, Ruiz-Cabello F, et al. (2007) Role of altered expression of HLA class I molecules in cancer progression. Adv Exp Med Biol 601: 123-131.

54. Kaneko K, Ishigami S, Kijima Y, Funasako Y, Hirata M, et al. (2011) Clinica implication of HLA class I expression in breast cancer. BMC Cancer 11: 454

55. Chang CC, Campoli M, Ferrone S (2003) HLA class I defects in malignant lesions: what have we learned? Keio J Med 52: 220-229.

56. Chen SJ, Liu YL, Sytwu HK (2012) Immunologic regulation in pregnancy: from mechanism to therapeutic strategy for immunomodulation. Clin Dev Immuno 2012: 258391

57. Klenke FM, Abdollahi A, Bertl E, Gebhard MM, Ewerbeck V, et al. (2007) Tyrosine kinase inhibitor SU6668 represses chondrosarcoma growth via antiangiogenesis in vivo. BMC Cancer 7: 49.

58. Leucci E, Patella F, Waage J, Holmstrøm K, Lindow M, et al. (2013) microRNA-9 targets the long non-coding RNA MALAT1 for degradation in the nucleus. Sci Rep 3: 2535.

59. Levine AM, Tulpule A, Quinn DI, Gorospe G , Smith DL, et al. (2006) Phase study of antisense oligonucleotide against vascular endothelial growth factor decrease in plasmavascular endothelial growth factor with potential clinical efficacy. J Clin Oncol. 24:1712-1719. 